\title{
Burst behavior due to the quasimode excited by stimulated Brillouin scattering in high-intensity laser-plasma interactions
}

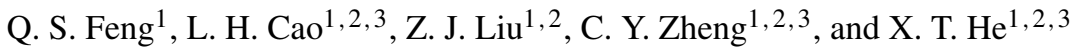 \\ ${ }^{1}$ Institute of Applied Physics and Computational Mathematics, Beijing 100094, China \\ ${ }^{2}$ HEDPS, Center for Applied Physics and Technology, Peking University, Beijing 100871, China \\ ${ }^{3}$ Collaborative Innovation Center of IFSA (CICIFSA), Shanghai Jiao Tong University, Shanghai 200240, China \\ (Received 21 April 2019; revised 23 July 2019; accepted 2 September 2019)
}

\begin{abstract}
The strong-coupling mode, called the "quasimode", is excited by stimulated Brillouin scattering (SBS) in high-intensity laser-plasma interactions. Also SBS of the quasimode competes with SBS of the fast mode (or slow mode) in multiion species plasmas, thus leading to a low-frequency burst behavior of SBS reflectivity. Competition between the quasimode and the ion-acoustic wave (IAW) is an important saturation mechanism of SBS in high-intensity laser-plasma interactions. These results give a clear explanation of the low-frequency periodic burst behavior of SBS and should be considered as a saturation mechanism of SBS in high-intensity laser-plasma interactions.
\end{abstract}

Keywords: Brillouin amplification; inertial confinement fusion; ion-acoustic waves; quasimode; stimulated Brillouin scattering

\section{Introduction}

Backward stimulated Brillouin scattering (SBS), a threewave interaction process where an incident electromagnetic wave (EMW) decays into a backscattered EMW and a forward propagating ion-acoustic wave (IAW), leads to a great energy loss of the incident laser and is detrimental in inertial confinement fusion $(\mathrm{ICF})^{[1-3]}$. Therefore, SBS plays an important role in the successful ignition goal of ICF. Multiple ion species are contained in the laser fusion program ${ }^{[4]}$. In indirect-drive $\mathrm{ICF}^{[2,3]}$ or hybrid-drive ignition $^{[1]}$, the inside of the hohlraum is filled with low$Z$ plasmas, such as $\mathrm{H}$ or $\mathrm{CH}$ plasmas from the initial filled material or from the material ablated off the capsule. Typically, in hybrid-drive ICF, the laser intensity can reach as high as $I_{0} \sim 10^{16} \mathrm{~W} / \mathrm{cm}^{2}$, and strong-coupling modes are excited by SBS. The stimulated scattering process in this regime is referred to as SBS in the strong-coupling regime or in the quasimode regime ${ }^{[5,6]}$. The quasimode or the strong-coupling mode refers to the modified lowfrequency mode involved in the three-wave process, while the electrostatic mode refers to a natural mode of the system

Correspondence to: L. H. Cao and C. Y. Zheng, No. 2, Fenghao East Road, Haidian District, Beijing 100094, China. Email: cao_lihua@iapcm.ac.cn (L. H. Cao), zheng_chunyang@iapcm.ac.cn (C. Y. Zheng) in the absence of the pump wave. The burst behavior of SBS reflectivity is universal in high-intensity laser-plasma interactions; however, the cause is not clear and requires explanation. Also, understanding the excitation of different IAW modes and competition between SBS of different modes under the condition of high-intensity laser-plasma interactions is vital to predict SBS laser energy losses and to improve energy coupling into the fusion capsule.

Besides SBS in ICF, the mechanism of SBS in the strongcoupling regime can also be applied to Brillouin amplification $^{[7-12]}$. Andreev et al. ${ }^{[8]}$ found that the interaction process of short light pulse amplification took place in the strongcoupling regime and that Brillouin scattering in the strongcoupling regime was a very promising candidate for the amplification of short laser pulses to high intensities over very short plasma lengths. Weber et al. ${ }^{[9]}$ demonstrated an optimum window where a Brillouin scheme could be exploited for amplification and compression of short laser pulses over short distances to very high power. Schluck et $a .^{[10]}$ investigated seed growth in the strong-coupling Brillouin case and discussed mitigation of spontaneous Raman scattering of the pump by the chosen chirp. Tsidulko et al. ${ }^{[12]}$ found that the precursors, growing from the leading part of the Raman-pumped seed pulse, could disturb the plasma and the pump ahead of the pumped pulses, which 
would jeopardize the promising scheme for producing ultrapowerful laser pulses through Raman backscattering of pump lasers in plasmas. In our work, the SBS in the strongcoupling regime would be used to amplify the seed pulses, especially the tail of the seed, as was demonstrated by Jia et al. ${ }^{[11]}$. Since the spontaneous SBS in this work grows very slowly, the spontaneous SBS cannot completely deplete the pump or ruin the plasma inhomogeneity before reaching the seed.

Many mechanisms for the saturation of SBS have been proposed, including frequency detuning due to particle trapping ${ }^{[13]}$, coupling with higher harmonics ${ }^{[14,15]}$, increasing linear Landau damping by kinetic ion heating ${ }^{[16,17]}$, the creation of cavitons in plasmas ${ }^{[18,19]}$, and so on. However, the burst behavior of SBS in high-intensity laser-plasma interactions is confusing and has not been explained well, which may be a potential saturation mechanism of SBS.

In this paper, we report the first demonstration that the strong-coupling mode or quasimode is excited by SBS and coexists and competes with the IAW in high-intensity laserplasma interactions. The competition between SBS of the IAW and SBS of the quasimode leads to a low-frequency burst behavior of SBS reflectivity and decreases the total SBS reflectivity. Therefore, competition between the quasimode and the IAW excited by SBS is an important saturation mechanism of SBS in high-intensity laser-plasma interactions.

\section{Theoretical analysis}

The wave number of the IAW excited by backward SBS can be calculated by

$$
k_{A} \lambda_{D e} \simeq 2 \frac{v_{t e}}{c} \sqrt{n_{c} / n_{e}-1},
$$

where $v_{t e}=\sqrt{T_{e} / m_{e}}$ is the electron thermal velocity, and $n_{e}, T_{e}$ and $m_{e}$ are the density, temperature and mass of the electron, respectively. Considering fully ionized, neutral, unmagnetized plasmas with the same temperature of all ion species $\left(T_{H}=T_{C}=T_{i}\right)$, the linear dispersion relation of the IAW in multi-ion species plasmas is given by ${ }^{[20-22]}$

$$
\epsilon\left(\omega, k=k_{A}\right)=1+\chi_{e}+\sum_{\beta} \chi_{i \beta}=0
$$

where $\chi_{e}$ is the susceptibility of the electron and $\chi_{i \beta}$ is the susceptibility of the ion $\beta$.

When strong pump light interacts with plasmas, the strong-coupling mode is generated, which grows with time and is not damped, and is called the quasimode. The strongcoupling regime of SBS is characterized by $\left(v_{o s} / v_{t e}\right)^{2}>$ $4 k_{0} c_{s} \omega_{0} / \omega_{p e}^{2}$, where $v_{t e}=\sqrt{T_{e} / m_{e}}, \omega_{p e}=\sqrt{4 \pi n_{e} e^{2} / m_{e}}$ and $v_{o s}=e E_{0} / m_{e} \omega_{0}$ are the electron thermal velocity,

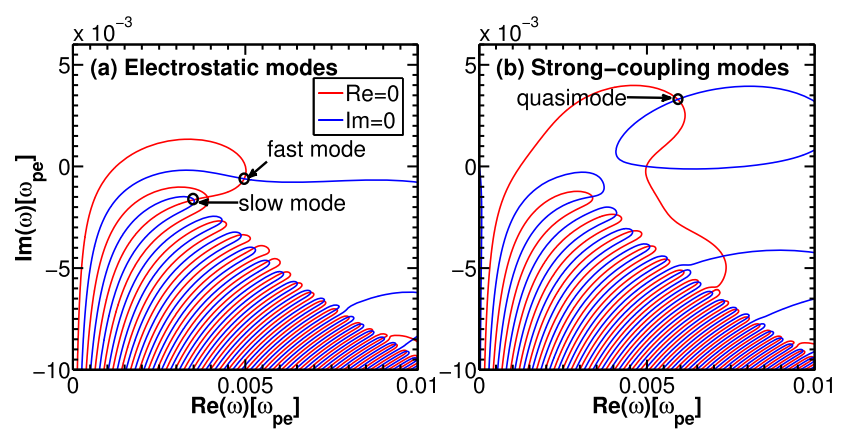

Figure 1. Contours of solutions to the dispersion relations of (a) the fast IAW mode and the slow IAW mode without pump light and (b) the quasimode with strong pump light $I_{0}=1 \times 10^{16} \mathrm{~W} / \mathrm{cm}^{2}$. The red line is $\operatorname{Re}[\epsilon]=0$ and the blue line is $\operatorname{Im}[\epsilon]=0$. The conditions are $T_{e}=5 \mathrm{keV}$, $T_{i}=0.2 T_{e}, n_{e}=0.3 n_{c}$ and $k_{A} \lambda_{D e}=0.3$ in a $\mathrm{C}_{2} \mathrm{H}$ plasma.

electron plasma frequency and electron quiver velocity, respectively. In this paper, strong laser refers to that with an intensity satisfying the condition of the strong-coupling regime of SBS. The dispersion relation of the quasimode in strong pump light is given by ${ }^{[23,24]}$

$$
\epsilon\left(\omega_{A}, k_{A}\right)=\frac{k_{A}^{2} v_{o s}^{2}}{4} \chi_{e}\left(1+\sum_{\beta} \chi_{i \beta}\right)\left(\frac{1}{D^{-}}+\frac{1}{D^{+}}\right),
$$

where $D^{-} \equiv D\left(\omega_{A}-\omega_{0}, k_{A}-k_{0}\right), D^{+} \equiv D\left(\omega_{A}+\omega_{0}, k_{A}+\right.$ $\left.k_{0}\right)$ and $D(\omega, k)=k^{2} c^{2}+\omega_{p e}^{2}-\omega^{2}$.

Under the condition $T_{e}=5 \mathrm{keV}$ and $n_{e}=0.3 n_{c}$, one can obtain the wave number of the IAW, $k_{A} \lambda_{D e}=0.3$, from Equation (1). By solving Equations (2) and (3), the contours of solutions of the IAW without pump light and with strong pump light are shown in Figure 1 under the condition $T_{i}=0.2 T_{e}$ and $k_{A} \lambda_{D e}=0.3$. Although there are infinite solutions of the IAW, as shown in Figure 1, the mode with the least Landau damping $(|\operatorname{Im}(\omega)|)$ is preferentially excited in SBS. There exist two groups of modes, called the 'fast mode' and the 'slow mode', in multi-ion species plasmas, as shown in Figure 1(a). Here, the fast mode and the slow mode refer to the least damped mode belonging to each class of mode. In the condition $T_{e}=5 \mathrm{keV}, T_{i}=0.2 T_{e}$ and $n_{e}=0.3 n_{c}$ in $\mathrm{C}_{2} \mathrm{H}$ plasmas, the laser intensity in the strong-coupling regime is $I_{0}>I_{s c}=1.98 \times 10^{15} \mathrm{~W} / \mathrm{cm}^{2}$. When strong pump light with an intensity greater than $I_{s c}=$ $1.98 \times 10^{15} \mathrm{~W} / \mathrm{cm}^{2}$ interacts with plasmas, the quasimode is excited. As shown in Figure 1(b), the imaginary part of the quasimode frequency is positive, which illustrates that the quasimode is a growing mode.

\section{Numerical simulation}

A one dimension in space and one dimension in velocity (1D1V) Vlasov-Maxwell code ${ }^{[25]}$ is used to research the 


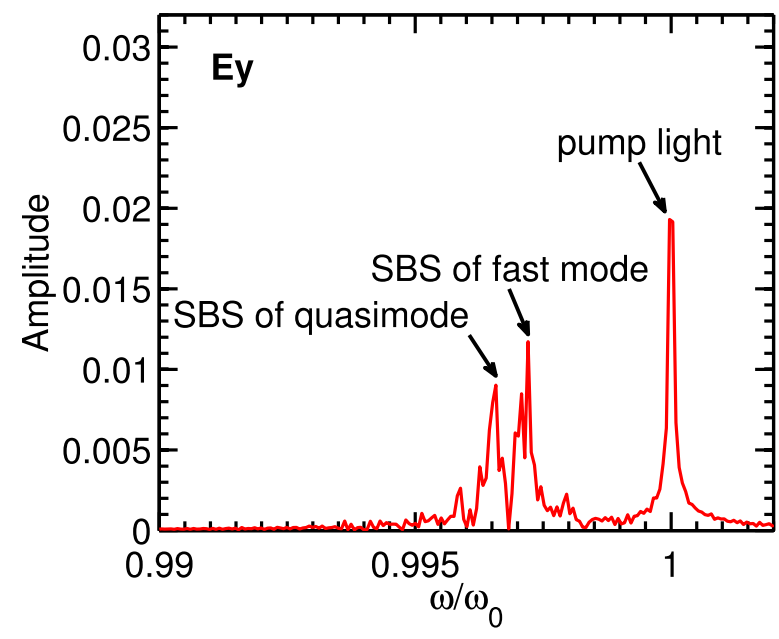

Figure 2. Frequency spectrum of $E_{y}$ with the time range $t \in[0,1 \times$ $\left.10^{5}\right] \omega_{0}^{-1}$ at $x_{0}=25 c / \omega_{0}$. The parameters are $n_{e}=0.3 n_{c}, T_{e}=$ $5 \mathrm{keV}, T_{i}=0.2 T_{e}$ and $I_{0}=1 \times 10^{16} \mathrm{~W} / \mathrm{cm}^{2}$ in a $\mathrm{C}_{2} \mathrm{H}$ plasma, the same as in Figure 1(b).

quasimode excited by SBS in multi-ion species plasmas. We choose the high-temperature and high-density region as an example: the electron temperature and electron density are $T_{e}=5 \mathrm{keV}$ and $n_{e}=0.3 n_{c}$, where $n_{c}$ is the critical density for the incident laser. The electron density is taken to be higher than $0.25 n_{c}$ - thus stimulated Raman scattering ${ }^{[26-28]}$ and two-plasmon decay instability ${ }^{[29,30]}$ are excluded. We take $\mathrm{C}, \mathrm{C}_{2} \mathrm{H}, \mathrm{CH}$, and $\mathrm{H}$ plasmas as typical examples since they are common in $\mathrm{ICF}^{[1,3]}$. The ion temperature is $T_{i}=0.2 T_{e}$. The linearly polarized laser intensity is $I_{0}=$ $1 \times 10^{16} \mathrm{~W} / \mathrm{cm}^{2}$, with wavelength $\lambda_{0}=0.351 \mu \mathrm{m}$, and the intensity of the seed light from the left boundary is $I_{s}=$ $10^{-6} I_{0}=1 \times 10^{10} \mathrm{~W} / \mathrm{cm}^{2}$ with frequency $\omega_{s}=0.994 \omega_{0}$. The spatial scale is $\left[0, L_{x}\right]$, discretized with $N_{x}=5000$ spatial grid points and a spatial step $\mathrm{d} x=0.2 c / \omega_{0}$. The spatial length is $L_{x}=1000 c / \omega_{0} \simeq 160 \lambda_{0}$ with $2 \times 5 \% L_{x}$ vacuum layers and $2 \times 5 \% L_{x}$ collision layers on the two sides of the plasma boundaries. The plasmas located at the center, with a density scale length $L=0.8 L_{x}$, are collisionless. The incident laser propagates along the $x$ axis from left to right with outgoing boundary conditions. Strong collision damping layers are added into the two sides of the plasma boundaries $\left(2 \times 5 \% L_{x}\right)$ to damp electrostatic waves, such as IAWs at the boundaries, and decrease the effect of the sheath field. The electron velocity scale $[-0.8 c, 0.8 c]$ and the ion velocity scale $[-0.03 c, 0.03 c]$ are discretized with $2 N_{v}+1\left(N_{v}=512\right)$ grid points. The total simulation time is $t_{\text {end }}=1 \times 10^{5} \omega_{0}^{-1}$, discretized with $N_{t}=5 \times 10^{5}$ and a time step $\mathrm{d} t=0.2 \omega_{0}^{-1}$.

Figure 2 shows the spectra of the SBS scattered light in the case of $\mathrm{C}_{2} \mathrm{H}$ plasmas. Calculated by the Vlasov simulation, the two peak frequencies of scattered light in $\mathrm{C}_{2} \mathrm{H}$ plasmas are $\omega_{s}=0.9972 \omega_{0}, 0.9966 \omega_{0}$. Calculated from Equation (2)

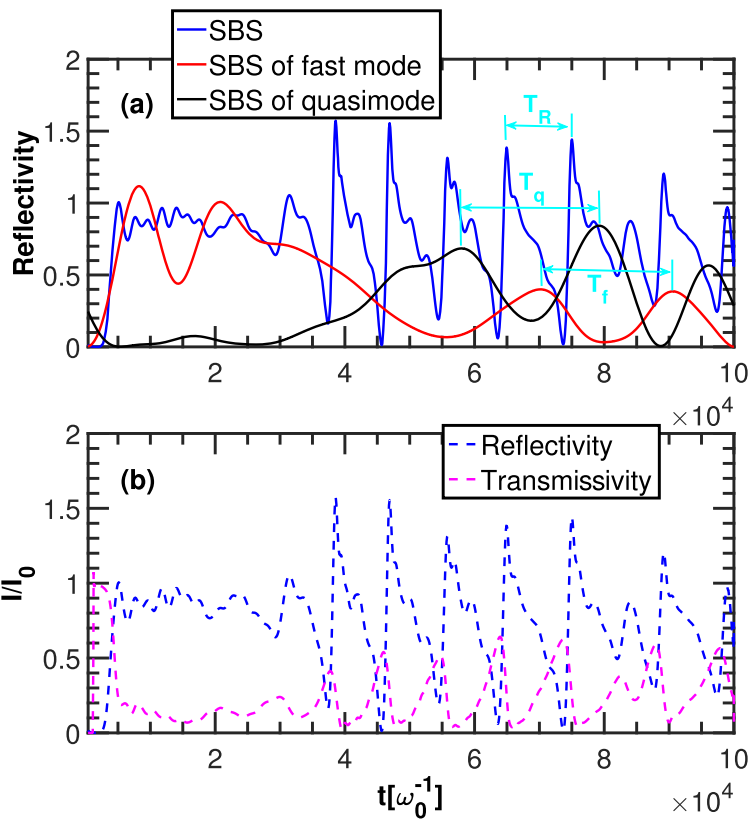

Figure 3. (a) Evolution of the SBS reflectivities of different modes with time, where SBS is the total SBS with the frequency range $\omega \in\left[0.9 \omega_{0}, 0.999 \omega_{0}\right]$, SBS of the fast mode with range $\omega \in$ $\left[0.9968 \omega_{0}, 0.9977 \omega_{0}\right]$ and SBS of the quasimode with range $\omega \in$ $\left[0.9960 \omega_{0}, 0.9968 \omega_{0}\right]$. (b) Reflectivity and transmissivity of the total SBS. The condition is the same as in Figure 2.

Table 1. Frequencies of different modes and the corresponding scattered light. The conditions are $T_{e}=5 \mathrm{keV}, T_{i}=0.2 T_{e}$, $n_{e}=0.3 n_{c}, k_{A} \lambda_{D e}=0.3$ and $I_{0}=1 \times 10^{16} \mathrm{~W} / \mathrm{cm}^{2}$ in $\mathrm{C}_{2} \mathrm{H}$ plasmas.

\begin{tabular}{lccc}
\hline & \multicolumn{2}{c}{ Theory } & Simulation \\
\cline { 2 - 3 } Mode & $\omega_{A} /\left(10^{-3} \omega_{0}\right)$ & $\omega_{S} / \omega_{0}$ & $\omega_{S} / \omega_{0}$ \\
\hline Fast mode & 2.7 & 0.9973 & 0.9972 \\
Slow mode & 1.9 & 0.9981 & \\
Quasimode & 3.8 & 0.9962 & 0.9966 \\
\hline
\end{tabular}

and Equation (3), the frequency of the fast mode is $\omega_{f}=$ $4.98 \times 10^{-3} \omega_{p e}=2.73 \times 10^{-3} \omega_{0}$, the frequency of the slow mode is $\omega_{s}=3.49 \times 10^{-3} \omega_{p e}=1.9 \times 10^{-3} \omega_{0}$, and the frequency of the quasimode with pump light $I_{0}=$ $1 \times 10^{16} \mathrm{~W} / \mathrm{cm}^{2}$ is $\omega_{q}=6.98 \times 10^{-3} \omega_{p e}=3.82 \times 10^{-3} \omega_{0}$. Therefore, the corresponding theoretical frequencies of SBS scattered light of the fast mode, the slow mode and the quasimode are $\omega_{s}=0.9973 \omega_{0}, 0.9981 \omega_{0}, 0.9962 \omega_{0}$. The theoretical and simulation results are shown in Table 1. Compared to the theoretical frequencies, we can see that the two peaks of the SBS scattered light in Figure 2 are indeed the SBS of the fast mode and the SBS of the quasimode.

Figure 3 gives the evolution of the SBS reflectivities of the fast mode and the quasimode with time. As shown in Figure 3(a), the SBS of the fast mode develops quickly before $\sim 1 \times 10^{4} \omega_{0}^{-1}$ and saturates during $t \in\left[1 \times 10^{4}, 3 \times\right.$ $\left.10^{4}\right] \omega_{0}^{-1}$. In this stage, the entire SBS reflectivity (blue line 

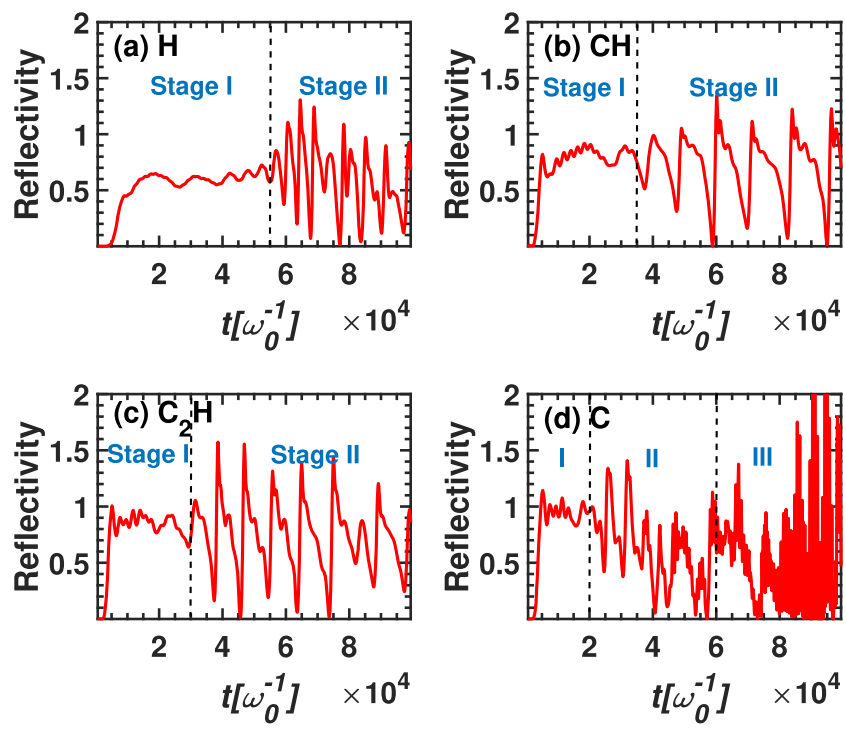

Figure 4. Evolution of the SBS reflectivities in different species plasmas with time.

in Figure 3(a)) is from the SBS of the fast mode. After $3 \times$ $10^{4} \omega_{0}^{-1}$, SBS of the quasimode develops and competes with the SBS of the fast mode, which leads to the burst behavior of the total SBS reflectivity. The SBS of the fast mode exhibits an obvious decrease when the SBS of the quasimode develops to the peak amplitude during $\left[3 \times 10^{4}, 6 \times 10^{4}\right] \omega_{0}^{-1}$. After $6 \times 10^{4} \omega_{0}^{-1}$, there is a contradictory trend between the SBS of the fast mode and the SBS of the quasimode, which illustrates the competition between the corresponding SBS of the two modes. The recurrence period of the total SBS reflectivity is $T_{R}=7.501 \times 10^{4} \omega_{0}^{-1}-6.494 \times 10^{4} \omega_{0}^{-1}=$ $1.007 \times 10^{4} \omega_{0}^{-1}$, as calculated from the two peaks of the SBS reflectivity shown in Figure 3(a); thus the recurrence frequency of SBS reflectivity is $\omega_{R}=1 / T_{R}=9.93 \times$ $10^{-5} \omega_{0}^{-1}$. In the same way, the period of the SBS of the fast mode is $T_{f}=2.031 \times 10^{4} \omega_{0}^{-1}$ and the period of the SBS of the quasimode is $T_{q}=2.126 \times 10^{4} \omega_{0}^{-1}$. Thus, the sum of the two periods is $\omega_{f}+\omega_{q}=1 / T_{f}+1 / T_{q}=9.63 \times 10^{-5} \omega_{0}^{-1}$, which is close to the recurrence period of the SBS reflectivity $T_{R}$. This illustrates that the burst behavior of the SBS reflectivity is a result of competition between the SBS of the fast mode and the SBS of the quasimode, and the recurrence period of the SBS reflectivity burst comes from the sum of the periods of the SBS of the fast mode and the SBS of the quasimode. Figure 3(b) demonstrates the total reflectivity and transmissivity of the SBS. Since the burst behavior of the total reflectivity of SBS occurs due to competition between SBS of the fast mode and SBS of the quasimode, the transmissivity demonstrates the same burst behavior, which is complementary to the SBS reflectivity after $3 \times 10^{4} \omega_{0}^{-1}$. Therefore, competition between SBS of the fast mode and SBS of the quasimode is an important factor in the burst behavior of SBS and the nonlinear saturation of SBS.
Figure 4 demonstrates the SBS reflectivities in different species plasmas. In stage I, the SBS reflectivities develop and then saturate at a nearly fixed level. The saturation time of the SBS of the electrostatic IAW mode can be calculated from $t_{\text {sat }}=\ln \left(I_{\text {sat }} / I_{S}\right) / \gamma_{0 B}$, where $\gamma_{0 B}$ is the SBS maximum temporal growth rate, which is defined in Equation (7). Taking $\mathrm{C}_{2} \mathrm{H}$ as an example, $\gamma_{0 B}=2.2 \times 10^{-3} \omega_{0}$ from Equation (7); thus, the saturation time of the scattering from $I_{S}=1 \times 10^{-6} I_{0}$ to $I_{\mathrm{sat}} \simeq I_{0}$ is $t_{\mathrm{sat}}=\ln \left(I_{\mathrm{sat}} / I_{S}\right) / \gamma_{0 B} \simeq$ $\ln \left(1 / 10^{-6}\right) /\left(2.2 \times 10^{-3} \omega_{0}\right)=6.28 \times 10^{3} \omega_{0}^{-1}$. From Figure 3(a), the saturation time of the SBS of the fast mode with a reflectivity of approximately 1 is $t_{\text {sat }} \simeq 6.72 \times 10^{3} \omega_{0}^{-1}$, which is close to the prediction of the linear theoretical model. In stage II, when different modes, such as the fast IAW mode and the quasimode, are excited in SBS in our simulation conditions, other modes such as the slow IAW mode and the ion bulk (IBk) mode ${ }^{[31]}$ could be excited in SBS in some conditions, and the competition among SBS of different modes leads to the low-frequency burst behavior of SBS reflectivity. As the ratio of $\mathrm{C}$ to $\mathrm{H}$ increases, from Figure 4(a) to Figure 4(d), the Landau damping of the IAW decreases and the SBS growth rate and SBS reflectivity increase. The saturation time $t_{\text {sat }}$ becomes shorter. When the SBS of the electrostatic IAW saturates, the IAW amplitude no longer increases and the quasimode starts to develop, as shown in Figure 3(a), which leads to competition between the quasimode and the IAW and thereby the burst behavior of SBS reflectivity. The earlier the SBS of the electrostatic IAW saturates, the earlier the burst behavior of SBS reflectivity occurs. As a result, the initial time of stage II will be earlier.

The burst period of SBS reflectivity $T_{R}$ is related to the maximum electrostatic field $E_{x}^{m}$ and the growth rate of SBS $\gamma_{0 B}$. The electrostatic field grows from the initial noise level $E_{x}^{0}$ to the maximum electrostatic field $E_{x}^{m}$ in about half the burst period $T_{R} / 2$, satisfying the relation

$$
\left|E_{x}^{m}\right|^{2}=\left|E_{x}^{0}\right|^{2} \times \exp \left(\gamma_{0 B} \times T_{R} / 2\right) .
$$

Thus, the approximate relation of the burst period can be expressed as

$$
T_{R}=\frac{4}{\gamma_{0 B}} \ln \frac{E_{x}^{m}}{E_{x}^{0}},
$$

where the maximum electrostatic field $E_{x}^{m}$ is decided by the wave breaking of the IAW. Taking $\mathrm{C}_{2} \mathrm{H}$ plasma as an example, $e E_{x}^{m} /\left(m_{e} \omega_{0} c\right) \simeq 4 \times 10^{-3}$ can be obtained from the simulation. The noise electrostatic field $e \phi / T_{e}=\delta n / n=$ $\left(I_{s} / I_{0}\right)^{1 / 2}=10^{-3}$; thus, $e E_{x}^{0} /\left(m_{e} \omega_{p e} v_{t e}\right)=e \phi / T_{e} \times$ $\left(k \lambda_{D e}\right)=3 \times 10^{-4}$ and $e E_{x}^{0} /\left(m_{e} \omega_{0} c\right)=e E_{x}^{0} /\left(m_{e} \omega_{p e} v_{t e}\right) \times$ $\left(\omega_{\text {pe }} / \omega_{0}\right) \times\left(v_{t e} / c\right)=1.625 \times 10^{-5}$. As a result, one can calculate $T_{R} \simeq 1.001 \times 10^{4} \omega_{0}^{-1}$ from Equation (5), which is close to the burst period $T_{R}=1.007 \times 10^{4} \omega_{0}^{-1}$ from the Vlasov simulation. The maximum electrostatic field $E_{x}^{m}$ decided by the wave breaking of the IAW is related to the phase velocity of the IAW. In our simulation, the dominant 


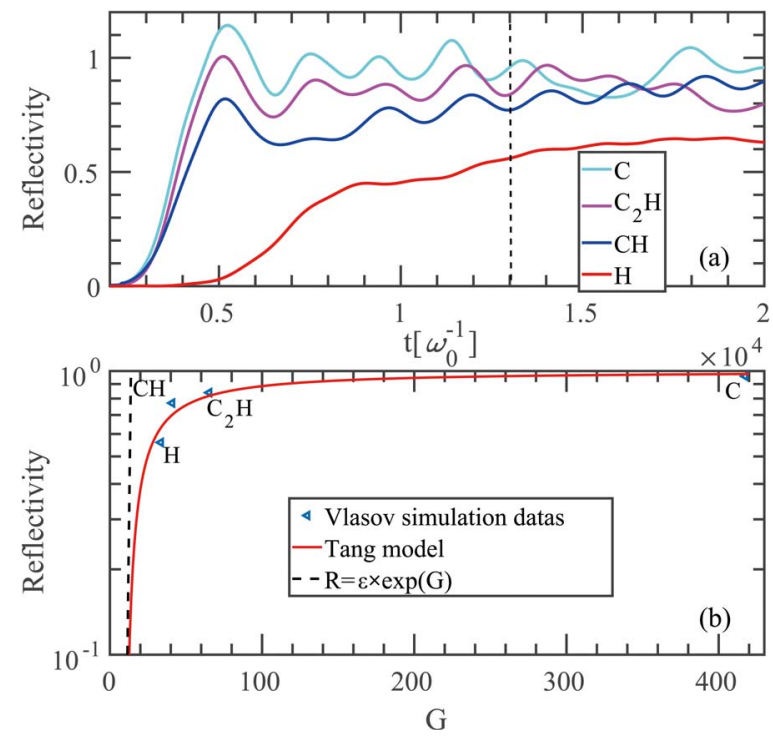

Figure 5. (a) Early linear stage of SBS in different species plasmas. (b) Relation between the SBS reflectivity and SBS gain in different species plasmas, where the gains in multi-ion species plasmas, such as $\mathrm{CH}$ and $\mathrm{C}_{2} \mathrm{H}$ plasmas, are calculated by the kinetic theory, and the gains in singleion species plasmas, such as $\mathrm{H}$ and $\mathrm{C}$ plasmas, are calculated by the fluid theory. The SBS reflectivities by the Vlasov simulation take the values at $t=1.3 \times 10^{4} \omega_{0}^{-1}$.

IAW is the fast mode. The phase velocity increases and the maximum electrostatic field $E_{x}^{m}$ decreases in the plasma sequence $\mathrm{CH}, \mathrm{C}_{2} \mathrm{H}, \mathrm{C}$ and $\mathrm{H}$. Thus, from $\mathrm{CH}, \mathrm{C}_{2} \mathrm{H}, \mathrm{C}$ to $\mathrm{H}$ plasmas in sequence, the burst period $T_{R}$ decreases, which is consistent with the results shown in Figure 4.

However, for $\mathrm{C}$ plasmas, the Landau damping of the IAW is very low and the gain of the SBS is very high in the condition $I_{0}=1 \times 10^{16} \mathrm{~W} / \mathrm{cm}^{2}$, and the SBS cascade occurs in stage III ${ }^{[32]}$. The low-frequency burst in stage II is due to the competition among SBS of different modes, while the high-frequency burst in stage III is a result of SBS cascade. These results in Figure 3 give a clear explanation of the low-frequency burst behavior in SBS, which is a common phenomenon of SBS in high-intensity laser-plasma interactions.

Figure 5(a) shows the linear growth and saturation process of the SBS in the early stage, in which the conditions are the same as in Figure 4. We can see that the SBS in C plasmas increases the most quickly among the four cases, while the growth rate of the SBS in $\mathrm{H}$ plasmas is the slowest and the saturation level is the lowest. As the ratio of $\mathrm{H}$ to $\mathrm{C}$ in the plasma increases, the growth rate and saturation level clearly decrease, because of the increase in the IAW Landau damping. According to Figure 5(a), the SBS reflectivity values at the time $t=1.3 \times 10^{4} \omega_{0}^{-1}$ are chosen as the saturation values, as shown in Figure 5(b), because they are stable at this time. The linear gain of SBS by fluid theory is given by

$$
G=2 \frac{\gamma_{0 B}^{2}}{v_{A} v_{g s}} L,
$$

where

$$
\gamma_{0 B}=\frac{1}{4} \sqrt{\frac{n_{e}}{n_{c}}} \frac{v_{0}}{v_{t e}} \sqrt{\omega_{0} \omega_{A}}
$$

is the maximum temporal growth rate of $\mathrm{SBS}^{[33,34]}$ and $v_{0}=e A_{0} / m_{e} c$ is the electron quiver velocity. $v_{g s}=c^{2} k_{s} / \omega_{s}$ is the group velocity of the SBS scattered light and $L$ is the plasma density scale length, while $\omega_{A} \equiv \operatorname{Re}\left(\omega_{A}\right)$ and $v_{A} \equiv\left|\operatorname{Im}\left(\omega_{A}\right)\right|$ are the frequency and Landau damping of the IAW. The collision damping of the IAW can be neglected since the electron temperature is as high as $T_{e}=5 \mathrm{keV}$ in our simulation; thus only the Landau damping of the IAW is considered. For single-ion species plasmas, such as $\mathrm{H}$ or $\mathrm{C}$ plasmas, the gain of SBS can be calculated by means of the fluid theory. However, for multi-ion species homogeneous plasmas, the SBS gain can be calculated using the kinetic theory ${ }^{[34]}$ :

$$
G\left(\omega_{s}\right)=\frac{1}{4} \frac{k_{A}^{2} v_{0}^{2} L}{v_{g s} \omega_{s}} \operatorname{Im}\left[\frac{\chi_{e}\left(1+\sum_{i} \chi_{i}\right)}{\epsilon\left(k_{s}-k_{0}, \omega_{s}-\omega_{0}\right)}\right],
$$

which is more precise than fluid theory. Here, subscripts $0, s, A$ represent the pump light, the SBS scattered light and the IAW. Under the strong damping condition $v_{A} / \gamma_{0 B} \times$ $\sqrt{v_{g s} / v_{g A}} \gg 1^{[35]}$, one obtains the Tang model ${ }^{[36]}$ :

$$
R(1-R)=\varepsilon\{\exp [G(1-R)]-R\},
$$

where $R$ is the reflectivity of SBS at the left boundary and $\varepsilon$ is the seed light at the right boundary. If $R \ll 1$, the Tang model can be approximated by the seed amplification equation:

$$
R=\varepsilon \times \exp (G)
$$

Although the strong damping condition in $\mathrm{C}$ plasmas or $\mathrm{C}_{2} \mathrm{H}$ plasmas is not satisfied, the Tang model can also be applied in predicting the SBS reflectivity in the linear saturation stage. As shown in Figure 5(b), the SBS reflectivities in different species plasmas are close to the Tang model. Also, the seed amplification equation cannot be applied to predict the SBS reflectivities in the condition of strong pump light, since the SBS reflectivity $R$ is not much lower than 1 . Figure 5 shows the linear process, including a linear growth and saturation process, in which a linear theory such as the Tang model is applicable. However, in a nonlinear process, such as stage II and stage III in Figure 4, burst behavior occurs with the nonlinear saturation mechanism of SBS that has been explained in this paper. 


\section{Discussion}

In our work, $I_{s} / I_{0}=10^{-6} \sim(\delta n / n)^{2}$, where $I_{s}, I_{0}$ and $\delta n / n$ are the intensities of the seed light and pump light and the density fluctuation level, respectively. The scattered radiation from the thermal noise $I_{t h}$ can be considered as the low-amplitude seed light $I_{s}$ in the Vlasov simulation. Thus, $\delta n / n \sim 10^{-3}$ in our simulation, which is close to the density fluctuations in real experiments. The critical plasma length ${ }^{[8]}$ of pump depletion is $L_{p l, \text { crit }} \sim\left(v_{g 0} / \gamma_{s c}\right) \ln |n / \delta n|$, where $\gamma_{s c}=\operatorname{Im}\left(\omega_{s c}\right)$ is the growth rate of the quasimode in the strong-coupling regime. Taking $\mathrm{C}_{2} \mathrm{H}$ in our simulation condition as an example, $\gamma_{s c}=1.63 \times 10^{-3} \omega_{0}$, thus $L_{p l, \text { crit }} \simeq$ $3.54 \times 10^{3} c / \omega_{0}$. The plasma length is $L_{x}=1000 c / \omega_{0}$ in our simulation, which is shorter than the critical plasma length of pump depletion $L_{p l, \text { crit }}$. Thus, the pump depletion can hardly occur before reaching the seed. From Figure 5(a), we can see that the SBS reflectivity is nearly zero at $\omega_{0} t=1000$ when the pump light reaches the left boundary. If the plasma length is greater than $L_{p l, \text { crit }}$, spontaneous SBS develops to a high level and depletes the pump light in reaching the seed light. If the seed light is of the same intensity as the pump light, the SBS in the strong-coupling regime amplifies the seed light, especially the tail of the seed light, as was shown by Jia et al. ${ }^{[11]}$.

In real experiments, other physical processes, such as filamentation instability ${ }^{[37,38]}$, inhomogeneous plasma density, and inhomogeneous plasma flow, may take place and suppress the SBS. However, the main physics are obtained by a 1D simulation, since the SBS backward scattering occurs along the direction of propagation of the pump light. Although the results are presented by a 1D simulation, the burst phenomenon shown in this paper will occur in real experiments. Through the particle-in-cell (PIC) simulation code OSIRIS ${ }^{[39]}$ and the fluid simulation code $\operatorname{HLIP}^{[40]}$, the analogous burst behavior can also occur when an inhomogeneous plasma density and an inhomogeneous plasma flow are considered $^{[41]}$.

\section{Summary}

In conclusion, the quasimode is excited by SBS in highintensity laser-plasma interactions, which competes with the IAW excited by SBS. The competition between SBS of the quasimode and SBS of the fast mode in $\mathrm{C}_{2} \mathrm{H}$ plasmas demonstrates that this competition mechanism is the cause of the low-frequency burst behavior of SBS. In different species plasmas, the same low-frequency burst behavior will occur, thus illustrating that the competition mechanism is common in high-intensity laser-plasma interactions no matter what the plasmas are. These results also give a good explanation of the intermediate low-frequency burst and saturation process in $\mathrm{C}$ plasmas.

\section{Acknowledgements}

We would like to acknowledge useful discussions with C. Z. Xiao and L. Hao. This research was supported by the National Natural Science Foundation of China (Nos. 11875091, 11575035, 11875093, 11475030 and 11435011), National Postdoctoral Program for Innovative Talents (No. BX20180055), China Postdoctoral Science Foundation (No. 2018M641274) and Science Challenge Project (No. TZ2016005).

\section{References}

1. X. T. He, J. W. Li, Z. F. Fan, L. F. Wang, J. Liu, K. Lan, J. F. Wu, and W. H. Ye, Phys. Plasmas 23, 082706 (2016).

2. S. H. Glenzer, B. J. MacGowan, P. Michel, N. B. Meezan, L. J. Suter, S. N. Dixit, J. L. Kline, G. A. Kyrala, D. K. Bradley, D. A. Callahan, E. L. Dewald, L. Divol, E. Dzenitis, M. J. Edwards, A. V. Hamza, C. A. Haynam, D. E. Hinkel, D. H. Kalantar, J. D. Kilkenny, O. L. Landen, J. D. Lindl, S. LePape, J. D. Moody, A. Nikroo, T. Parham, M. B. Schneider, R. P. J. Town, P. Wegner, K. Widmann, P. Whitman, B. K. F. Young, B. Van Wonterghem, J. Atherton, and E. I. Moses, Science 327, 228 (2010).

3. S. H. Glenzer, D. H. Froula, L. Divol, M. Dorr, R. L. Berger, S. Dixit, B. A. Hammel, C. Haynam, J. A. Hittinger, J. P. Holder, O. S. Jones, D. H. Kalantar, O. L. Landen, A. B. Langdon, S. Langer, B. J. MacGowan, A. J. Mackinnon, N. Meezan, E. I. Moses, C. Niemann, C. H. Still, L. J. Suter, R. J. Wallace, E. A. Williams, and B. K. F. Young, Nat. Phys. 3, 716 (2007).

4. P. Neumayer, R. L. Berger, L. Divol, D. H. Froula, R. A. London, B. J. MacGowan, N. B. Meezan, J. S. Ross, C. Sorce, L. J. Suter, and S. H. Glenzer, Phys. Rev. Lett. 100, 105001 (2008)

5. C. S. Liu, M. N. Rosenbluth, and R. B. White, Phys. Fluids 17, 1211 (1974).

6. P. N. Guzdar, C. S. Liu, and R. H. Lehmberg, Phys. Plasmas 3, 3414 (1996).

7. V. Malkin, G. Shvets, and N. J. Fisch, Phys. Rev. Lett. 82, 4448 (1999).

8. A. A. Andreev, C. Riconda, V. T. Tikhonchuk, and S. Weber, Phys. Plasmas 13, 053110 (2006).

9. S. Weber, C. Riconda, L. Lancia, J. R. Marques, G. A. Mourou, and J. Fuchs, Phys. Rev. Lett. 111, 055004 (2013).

10. F. Schluck, G. Lehmann, and K. H. Spatschek, Phys. Plasmas 22, 093104 (2015)

11. Q. Jia, I. Barth, M. R. Edwards, J. M. Mikhailova, and N. J. Fisch, Phys. Plasmas 23, 053118 (2016).

12. Yu. A. Tsidulko, V. M. Malkin, and N. J. Fisch, Phys. Rev. Lett. 88, 235004 (2002).

13. D. H. Froula, L. Divol, and S. H. Glenzer, Phys. Rev. Lett. 88 , 105003 (2002).

14. B. I. Cohen, B. F. Lasinski, A. B. Langdon, and E. A. Williams, Phys. Plasmas 4, 956 (1997).

15. W. Rozmus, M. Casanova, D. Pesme, A. Heron, and J. Adam, Phys. Fluids B 4, 576 (1992).

16. P. W. Rambo, S. C. Wilks, and W. L. Kruer, Phys. Rev. Lett. 79, 83 (1997).

17. C. J. Pawley, H. E. Huey, and N. C. Luhmann, Phys. Rev. Lett. 49, 877 (1982).

18. S. Weber, C. Riconda, and V. T. Tikhonchuk, Phys. Rev. Lett. 94, 055005 (2005).

19. S. Weber, C. Riconda, and V. T. Tikhonchuk, Phys. Plasmas 12, 043101 (2005) 
20. E. A. Williams, R. L. Berger, R. P. Drake, A. M. Rubenchik, B. S. Bauer, D. D. Meyerhofer, A. C. Gaeris, and T. W. Johnston, Phys. Plasmas 2, 129 (1995).

21. Q. S. Feng, C. Y. Zheng, Z. J. Liu, C. Z. Xiao, Q. Wang, and X. T. He, Phys. Plasmas 23, 082106 (2016).

22. Q. S. Feng, C. Z. Xiao, Q. Wang, C. Y. Zheng, Z. J. Liu, L. H. Cao, and X. T. He, Phys. Rev. E 94, 023205 (2016).

23. J. F. Drake, P. K. Kaw, Y. C. Lee, G. Schmid, C. S. Liu, and M. N. Rosenbluth, Phys. Fluids 17, 778 (1974).

24. Z. J. Liu, X. T. He, C. Y. Zheng, and Y. G. Wang, Chin. Phys. B 21, 015202 (2012).

25. Z. J. Liu, S. P. Zhu, L. H. Cao, C. Y. Zheng, X. T. He, and Y. Wang, Phys. Plasmas 16, 112703 (2009).

26. Q. S. Feng, C. Y. Zheng, Z. J. Liu, L. H. Cao, Q. Wang, C. Z. Xiao, and X. T. He, Phys. Plasmas 25, 092112 (2018).

27. Y. Zhao, Z. Sheng, S. Weng, S. Ji, and J. Zhu, High Power Laser Sci. Eng. 7, 20 (2019).

28. S. Weber and C. Riconda, High Power Laser Sci. Eng. 3, e51 (2015).

29. C. Z. Xiao, Z. J. Liu, D. Wu, C. Y. Zheng, and X. T. He, Phys. Plasmas 22, 052121 (2015).

30. C. Z. Xiao, Z. J. Liu, C. Y. Zheng, and X. T. He, Phys. Plasmas 23, 022704 (2016).

31. Q. S. Feng, C. Y. Zheng, Z. J. Liu, L. H. Cao, C. Z. Xiao, Q. Wang, H. C. Zhang, and X. T. He, Plasma Phys. Control. Fusion 59, 085007 (2017).
32. Q. S. Feng, Z. J. Liu, C. Y. Zheng, C. Z. Xiao, Q. Wang, H. C. Zhang, L. H. Cao, and X. T. He, Plasma Phys. Control. Fusion 59, 075007 (2017).

33. R. L. Berger, L. J. Suter, L. Divol, R. A. London, T. Chapman, D. H. Froula, N. B. Meezan, P. Neumayer, and S. H. Glenzer, Phys. Rev. E 91, 031103(R) (2015).

34. J. D. Lindl, P. Amendt, R. L. Berger, S. G. Glendinning, S. H. Glenzer, S. W. Haan, R. L. Kauffman, O. L. Landen, and L. J. Suter, Phys. Plasmas 11, 339 (2004).

35. D. W. Forslund, J. M. Kindel, and E. L. Lindman, Phys. Fluids 18, 1002 (1975).

36. C. L. Tang, J. Appl. Phys. 37, 2945 (1966).

37. T. W. Huang, C. T. Zhou, A. P. L. Robinson, B. Qiao, H. Zhang, S. Z. Wu, H. B. Zhuo, P. A. Norreys, and X. T. He, Phys. Rev. E 92, 053106 (2015).

38. V. Y. Bychenkov, W. Rozmus, A. V. Brantov, and V. T. Tikhonchuk, Phys. Plasmas 7, 1511 (2000).

39. R. Fonseca, L. Silva, F. Tsung, V. Decyk, W. Lu, C. Ren, W. Mori, S. Deng, S. Lee, T. Katsouleas, and J. C. Adam, Lect. Notes Comput. Sci. 2331, 342 (2002).

40. L. Hao, Y. Q. Zhao, D. Yang, Z. J. Liu, X. Y. Hu, C. Y. Zheng, S. Y. Zou, F. Wang, X. S. Peng, Z. C. Li, S. W. Li, T. Xu, and H. Y. Wei, Phys. Plasmas 21, 072705 (2014).

41. L. Hao, J. Li, W. D. Liu, R. Yan, and C. Ren, Phys. Plasmas 23, 042702 (2016). 